Supporting Information

\title{
The In Vitro Characterization of the Iterative Avermectin Glycosyltransferase AveBI Reveals Reaction Reversibility and Sugar Nucleotide Flexibility
}

\author{
Changsheng Zhang, Christoph Albermann, Xun Fu, and Jon S. Thorson*
}

Laboratory for Biosynthetic Chemistry, Pharmaceutical Sciences Division, School of Pharmacy, University of Wisconsin-Madison, 777 Highland Avenue, Madison, Wisconsin 53705

\section{Materials and Methods}

Materials. E. coli $\mathrm{DH} 5 \alpha$ and $\mathrm{BL} 21(\mathrm{DE} 3)$ competent cells were purchased from Invitrogen. The E. coli expression vectors pET-11a, pET28a were purchased from Novagen. The plasmids, pPWW49 and pPWW50, for expression in S. lividans, were generous gifts from Dr. Udo F. Wehmeier and Prof. Dr. Wolfgang Piepersberg (Bergische University, Wuppertal, Germany). Primers were ordered from Intergrated DNA Technology. Pfu DNA polymerase was purchased from Stratagene. Restriction enzymes and T4 DNA ligase were purchased from New England Biolabs. Other chemicals were purchased from Sigma (St. Louis, MO). TDP- $\alpha$-L-rhamnose and TDP- $\beta$-L-rhamnose were gifts from Dr. Svetlana Borisova and Prof. Dr. Hung-wen Liu (University of Texas at Austin, Austin, USA). Ivermectin was purchased from Sigma (St. Louis, MO) and avermectin B1a was purchased from Supelco (Bellefonte, PA). ${ }^{1} \mathrm{H}$ NMR, ${ }^{13} \mathrm{C}$ NMR and two-dimensional correlation spectra (gCOSY, TOCXY, gHSQC and gHMBC) were recorded in $\mathrm{CD}_{3} \mathrm{OD}$ on a $400-\mathrm{MHz} \mathrm{Varian}$ INOVA model NMR spectrometer. Chemical shifts are reported in parts per million (ppm, $\delta$ ) relative to $C D_{3} O D$ (0.00). ${ }^{1} \mathrm{H}$ NMR splitting patterns with observed first-order coupling are designated as singlet (s), doublet (d), or triplet (t). Splitting patterns that could not be interpreted or easily visualized are designated as multiplet (m). Mass spectra (MS) were obtained by using electrospray ionization on Agilent 1100 HPLC-MSD SL quadrupole mass spectometer connected with a UV/Vis diode array detector.

Chemoenzymatic Synthesis of TDP-sugars. The RmIA (glucose-1-phosphate thymidylyltransferase, sometimes also referred to as ' $E_{p}$ ') reaction was carried out in Tris- $\mathrm{HCl}$ buffer $(50 \mathrm{mM}, \mathrm{pH} 8.0)$ containing $5 \mathrm{mM}$ $\mathrm{MgCl}_{2}, 1 \mathrm{U}$ inorganic pyrophosphatase, $10 \mu \mathrm{M}$ of purified RmIA, $8 \mathrm{mM}$ sugar-1-phosphate and $6 \mathrm{mM}$ TTP, and incubated at $37^{\circ} \mathrm{C}$ for $2 \mathrm{~h}$. The formation of TDP-sugars was monitored by RP-HPLC (Phenomenex, Luna C18, $5 \mu \mathrm{m}, 250 \times 4.6 \mathrm{~mm}, 30 \mathrm{mM} \mathrm{KH}_{2} \mathrm{PO}_{4}, \mathrm{pH} 6.0,5 \mathrm{mM}$ tetrabutylammonium hydrogensulfate, $2 \% \mathrm{CH}_{3} \mathrm{CN}$ with a gradient of $0-50 \% \mathrm{CH}_{3} \mathrm{CN}$ over $30 \mathrm{~min}, 1 \mathrm{~mL} / \mathrm{min}, \mathrm{A}_{254}$ ). The enzymatic and/or chemical syntheses of all sugar nucleotides utilized in this study have been previously described. ${ }^{1}$

Preparation of Aglycons. Ivermectin (7, $460 \mathrm{mg}, 0.525 \mathrm{mmol}$ ) was added to a solution of $10 \mathrm{ml}_{2} \% \mathrm{H}_{2} \mathrm{SO}_{4}$ in isopropanol and stirred at room temperature under argon for $6 \mathrm{~h}$. The reaction was quenched via addition of $0.1 \mathrm{~mL}$ of triethylamine $\left(\mathrm{NEt}_{3}\right)^{2}{ }^{2}$ Sample was dried, dissolved in $500 \mu \mathrm{l}$ methanol and loaded onto a silica column $(3 \times 30 \mathrm{~cm})$ pre-equilibrated with petroleum. Elution with EtOAc/petroleum varying from 0/10, 1/9, 2/8, 3/7, 2/6 (ea. $100 \mathrm{ml}$ ) gave $5(125.9 \mathrm{mg}, 0.215 \mathrm{mmol}, 41 \%), 6(182.7 \mathrm{mg}, 0.250 \mathrm{mmol}, 48 \%)$ and a trace amount of $8(1 \mathrm{mg})$. Similarly, $2(8.9 \mathrm{mg}, 0.015 \mathrm{mmol}, 17 \%)$ and $3(39.0 \mathrm{mg}, 0.053 \mathrm{mmol}, 61 \%)$ were prepared from AVM B1a (1, $76 \mathrm{mg}, 0.087 \mathrm{mmol})$.

Compound 5. ${ }^{1} \mathrm{H}-\mathrm{NMR}\left(400 \mathrm{~Hz}, \mathrm{CD}_{3} \mathrm{OD}\right): \delta 3.26(\mathrm{~d}, \mathrm{~J}=1.8 \mathrm{~Hz}, 1 \mathrm{H}), 5.46(\mathrm{~d}, \mathrm{~J}=1.8 \mathrm{~Hz}, 1 \mathrm{H}), 1.86(\mathrm{~s}, 3 \mathrm{H}), 4.27(\mathrm{~d}, \mathrm{~J}=5.6$ $\mathrm{Hz}, 1 \mathrm{H}), 3.80$ (d, J=5.6 Hz, 1H), 4.63, 4.70 (d, J=14 Hz, 2H), 5.83 (d, J=11.2 Hz, 1H), 5.92 (dd, J=11.2, $14.8 \mathrm{~Hz}, 1 \mathrm{H}), 5.72$ (dd, J=14.8, $10 \mathrm{~Hz}, 1 \mathrm{H}), 2.63(\mathrm{~m}, 1 \mathrm{H}), 1.18(\mathrm{~d}, \mathrm{~J}=6.8 \mathrm{~Hz}, 3 \mathrm{H}), 3.99(\mathrm{br}, 1 \mathrm{H}), 1.57(\mathrm{~s}, 3 \mathrm{H}), 5.49(\mathrm{t}, J=8.0 \mathrm{~Hz}, 1 \mathrm{H}), 2.32(\mathrm{t}$, $J=8.0 \mathrm{~Hz}, 2 \mathrm{H}), 3.74(\mathrm{~m}, 1 \mathrm{H}), 0.85,1.95(\mathrm{~m}, 2 \mathrm{H}), 5.03(\mathrm{~m}, 1 \mathrm{H}), 2.24(\mathrm{dd}, J=4.0,12 \mathrm{~Hz}, 1 \mathrm{H}), 1.24(\mathrm{t}, J=12 \mathrm{~Hz}, 1 \mathrm{H}), 1.5-1.6$ $(\mathrm{m}, 5 \mathrm{H}), 0.85(\mathrm{~d}, J=5.6 \mathrm{~Hz}, 3 \mathrm{H}), 3.29(\mathrm{~m}, 1 \mathrm{H}), 1.55(\mathrm{~m}, 1 \mathrm{H}), 0.91(\mathrm{~d}, J=6.8 \mathrm{~Hz}, 3 \mathrm{H}), 1.48(\mathrm{~m}, 2 \mathrm{H}), 1.01(\mathrm{t}, J=7.4 \mathrm{~Hz}, 3 \mathrm{H})$; ${ }^{13} \mathrm{C}-\mathrm{NMR}\left(\mathrm{CD}_{3} \mathrm{OD}\right): \delta$ 173.47, 47.10, 120.30, 137.17, 19.93, 69.01, 82.26, 81.90, 141.49, 68.71, 121.91, 126.24, 138.38, 41.54, 19.94, 78.43, 140.46, 14.96, 118.40, 35.27, 69.14, 37.78, 70.20, 42.98, 98.96, 36.95(2C), 29.39, 18.01, 78.32, 32.59, 12.45, 28.65, 13.00. MS (m/z): calcd. 586.4, $[\mathrm{M}+\mathrm{H}]^{+} 587.2,[\mathrm{M}-\mathrm{H}]^{-} 585.4$. 
Compound 6. ${ }^{1} \mathrm{H}-\mathrm{NMR}\left(400 \mathrm{~Hz}, \mathrm{CD}_{3} \mathrm{OD}\right): \delta 3.25(\mathrm{~d}, \mathrm{~J}=1.8 \mathrm{~Hz}, 1 \mathrm{H}), 5.46(\mathrm{~d}, \mathrm{~J}=1.8 \mathrm{~Hz}, 1 \mathrm{H}), 1.85(\mathrm{~s}, 3 \mathrm{H}), 4.26(\mathrm{~d}, \mathrm{~J}=5.6 \mathrm{~Hz}$, $1 \mathrm{H}), 3.80(\mathrm{~d}, J=5.6 \mathrm{~Hz}, 1 \mathrm{H}), 4.62,4.67(\mathrm{~d}, J=14 \mathrm{~Hz}, 2 \mathrm{H}), 5.85(\mathrm{~d}, J=11.2 \mathrm{~Hz}, 1 \mathrm{H}), 5.91$ (dd, $J=11.2,14.8 \mathrm{~Hz}, 1 \mathrm{H}), 5.74(\mathrm{dd}$, $J=14.8,10 \mathrm{~Hz}, 1 \mathrm{H}), 2.68(\mathrm{~m}, 1 \mathrm{H}), 1.20(\mathrm{~d}, \mathrm{~J}=7.2 \mathrm{~Hz}, 3 \mathrm{H}), 4.02(\mathrm{br}, 1 \mathrm{H}), 1.57(\mathrm{~s}, 3 \mathrm{H}), 5.20(\mathrm{t}, \mathrm{J}=7.6 \mathrm{~Hz}, 1 \mathrm{H}), 2.33(\mathrm{t}, \mathrm{J}=7.6$ $\mathrm{Hz}, 2 \mathrm{H}), 3.74(\mathrm{~m}, 1 \mathrm{H}), 0.83,1.94(\mathrm{~m}, 2 \mathrm{H}), 5.05(\mathrm{~m}, 1 \mathrm{H}), 2.23(\mathrm{dd}, \mathrm{J}=4.0,12 \mathrm{~Hz}, 1 \mathrm{H}), 1.27(\mathrm{~m}, 1 \mathrm{H}), 1.5-1.6(\mathrm{~m}, 5 \mathrm{H}), 0.83(\mathrm{~d}$, $J=5.0 \mathrm{~Hz}, 3 \mathrm{H}), 3.31(\mathrm{~m}, 1 \mathrm{H}), 1.55(\mathrm{~m}, 1 \mathrm{H}), 0.91(\mathrm{~d}, J=6.4 \mathrm{~Hz}, 3 \mathrm{H}), 1.48(\mathrm{~m}, 2 \mathrm{H}), 0.99(\mathrm{t}, \mathrm{J}=7.4 \mathrm{~Hz}, 3 \mathrm{H}), 4.83(\mathrm{~d}, J=3.2 \mathrm{~Hz}$, $1 \mathrm{H}), 1.5(\mathrm{~m}, 2 \mathrm{H}), 3.55(\mathrm{~m}, \mathrm{~J}=9.2 \mathrm{~Hz}, 1 \mathrm{H}), 3.07(\mathrm{t}, J=9.4 \mathrm{~Hz}, 1 \mathrm{H}), 3.87(\mathrm{dd}, J=6.2,9.6 \mathrm{~Hz}, 1 \mathrm{H}), 1.25(\mathrm{~d}, J=6.2 \mathrm{~Hz}, 3 \mathrm{H}), 3.48$ (s, 3H); ${ }^{13} \mathrm{C}-\mathrm{NMR}\left(\mathrm{CD}_{3} \mathrm{OD}\right): \delta$ 173.58, 47.09, 120.29, 136.63, 19.94, 69.05, 82.27, 82.04, 141.94, 68.74, 121.76, 126.67, 138.39, 41.17, 20.98, 83.27, 137.26, 15.39, 120.03, 35.08, 69.03, 37.98, 70.27, 42.95, 99.08, 36.94(2C), 29.44, 17.97, $77.81,32.60,12.76,28.45,13.01,96.55,35.98,79.57,77.64,70.00,18.27,57.67 . \mathrm{MS}(\mathrm{m} / \mathrm{z}):$ calcd. $730.4,[\mathrm{M}+\mathrm{Na}]^{+} 753.0$, $[\mathrm{M}-\mathrm{H}]^{-} 729.4$.

The Cloning and overexpression of $a v e B I$ and purification of AveBI. The aveBI gene was amplified from pWHM473 using primers 5'- ctagacagtgacatatgtcagatcatttctcttc - 3' (forward, Ndel) and 5'aacctgtgagatctactcaccgcccggc - 3' (reverse, Bg/ll). The PCR products were digested with $N d e l / B g / l l$ and ligated into pPCPU21 (Ndel/Bg/ll) to give plasmid pCAM4.9. After confirmation by sequencing, the pCAM4.9 aveBI insert was subcloned into vectors pET11a, pET16b, pPWW49 and pPWW50 (Ndel/BamHI) ${ }^{3}$ to $\mathrm{provide}$ expression plasmids pCAM4.1, pCAM4.2, pCAM4.11 and pCAM4.10, respectively. Soluble expression of AveBI was only achieved in Streptomyces lividans TK64 harboring pCAM4.11 or pCAM4.10. Specifically, the plasmid pCAM4.10 or pCAM4.11 were introduced into S. lividans TK64 by standard transformation, ${ }^{4}$ transformants were grown in liquid TSB media (thiostrepton $25 \mu \mathrm{g} / \mathrm{mL}$ ) for 3 days at $28^{\circ} \mathrm{C}$ and were transferred to YEME media (thiostrepton $25 \mu \mathrm{g} / \mathrm{mL}$ ) containing $25 \%$ sucrose. The culture was incubated at $28^{\circ} \mathrm{C}$ for two more days and cells were harvested. For purification of the $N$-(His) $)_{6}$-tagged AveBI (pCAM4.10), cell pellets obtained from $300 \mathrm{~mL}$ of culture were washed twice with buffer $\mathrm{A}\left(20 \mathrm{mM} \mathrm{NaH} \mathrm{PO}_{4}, \mathrm{pH} 7.5,500 \mathrm{mM} \mathrm{NaCl}, 10\right.$ $\mathrm{mM}$ imidazole) and resuspended in $30 \mathrm{~mL}$ of buffer $A$ supplemented with $1 \mathrm{mg} / \mathrm{ml}$ of lysozyme. After $10 \mathrm{~min}$ incubation on ice, complete cell lysis was achieved by 3 rounds of French-press (1,200 psi, Thermo IEC) and the insoluble material was removed by centrifugation at $30,000 \mathrm{~g}$ for $1 \mathrm{hr}\left(4^{\circ} \mathrm{C}\right)$. The supernatant was loaded onto a HisTrap HT column $(1 \mathrm{~mL}$, Amersham Biosciences) and the $\mathrm{N}$-(His)-tagged AveBl eluted with a linear gradient of imidazole $(10-500 \mathrm{mM})$ in buffer A using a FPLC system (Amersham Biosciences). The purified protein was desalted through PD-10 column (Amersham Biosciences) and stored in the buffer containing 25 $\mathrm{mM}$ Tris- $\mathrm{HCl}(\mathrm{pH} 8.0), 100 \mathrm{mM} \mathrm{NaCl}$ and $10 \%$ glycerol until use. Protein concentration was measured by Bradford assay. ${ }^{5}$

AveBI assays. Generally, AveBI assays were performed in a total volume of $100 \mu \mathrm{L}$ in Tris-HCl buffer $(50 \mathrm{mM}$, $\mathrm{pH}$ 8.0) containing $2 \mathrm{mM} \mathrm{MgCl}_{2}$. Reversibility of AveBI reaction was assayed by co-incubation of avermectin B1a $(1,100 \mu \mathrm{M})$ and TDP $(2 \mathrm{mM})$ with $12 \mu \mathrm{M}$ AveBI at $30^{\circ} \mathrm{C}$ overnight. The AveBl-catalyzed aglycon exchange reaction was assayed by co-incubation of $100 \mu \mathrm{M} \mathbf{1}, 100 \mu \mathrm{M} 5$ and $2 \mathrm{mM}$ TDP with $12 \mu \mathrm{M}$ AveBI at $30^{\circ} \mathrm{C}$ overnight. To probe AveBI sugar substrate specificity, each reaction contained $50 \mu \mathrm{M}$ algycon (1-3, 5-8) and approximately $300 \mu \mathrm{M}$ TDP-sugar (directly from crude RmIA-catalyzed reactions) in the presence of $12 \mu \mathrm{M} \mathrm{AveBI}$ and incubated at $30^{\circ} \mathrm{C}$ overnight. The reactions were analyzed by reverse phase HPLC (Phenomenex Luna C18, $5 \mu \mathrm{m}, 250 \times 4.6 \mathrm{~mm}$; solvent $\mathrm{A}, 0.1 \% \mathrm{TFA}$ in water; solvent $\mathrm{B}, \mathrm{CH}_{3} \mathrm{CN}$; elution profile $-30 \%$ B to $70 \%$ B, 0-5 min; $70 \%$ B to $100 \%$ B, $5-25 \mathrm{~min} ; 100 \%$ B, $25-30 \mathrm{~min} ; 100 \%$ B to $30 \%$ B, 30-31 min and 30\%B, 31-40 min; $\left.1 \mathrm{~mL} / \mathrm{min}, \mathrm{A}_{243}\right)$.

\section{References}

1. (a) Barton, W. A.; Biggins, J. B.; Jiang, J.; Thorson, J. S.; Nikolov, D. B. Proc. Natl. Acad. Sci. U.S.A. 2002, 99, 13397-13402. (b) Jiang, J.; Biggins, J. B.; Thorson, J. S. Angew. Chem. Int. Ed. Engl. 2001, 40, 15021505. (c) Barton, W. A.; Lesniak, J.; Biggins, J. B.; Jeffrey, P. D.; Jiang, J.; Rajashankar, K. R.; Thorson, J. S.; Nikolov, D. B. Nat. Struct. Biol. 2001, 8, 545-551. (d) Jiang J.; Biggins J.B.; Thorson J. S. J. Am. Chem. Soc. 2000, 122, 6803-6804. (e) Albermann, C.; Soriano, A.; Jiang, J.; Vollmer, H.; Biggins, J. B.; Barton, W. A.; Lesniak, J.; Nikolov, D. B.; Thorson, J. S. Org. Lett. 2003, 5, 933-936. (f) Fu, X.; Albermann, C.; Jiang, J.; Liao, J.; Zhang, C.; Thorson, J. S. Nat. Biotechnol. 2003, 21, 1467-1469. (g) Borisova, S. A.; 
Zhang, C.; Takahashi, H.; Zhang, H.; Wong, A. W.; Thorson, J. S.; Liu, H. W. Angew. Chem. Int. Ed. 2006, 45, 2748-2753.

2. Wei, G. H.; Du, Y. G.; Linhardt, R. J. Tetrahedron. Lett. 2004, 45, 6895.

3. Doumith, M.; Weingarten, P.; Wehmeier, U. F.; Salah-Bey, K.; Benhamou, B.; Capdevila, C.; Michel, J. M.; Piepersberg, W.; Raynal, M. C. Mol. Gen. Genet. 2000, 264, 477.

4. Kieser, T.; Bibb, M. J.; Buttner, M. J.; Chater, K. F.; Hopwood, D. A., Practical Streptomyces Genetics. The John Innes Foundation: Norwich, England, 2000.

5. Bradford, M. M. Anal. Biochem. 1976, 72, 248. 


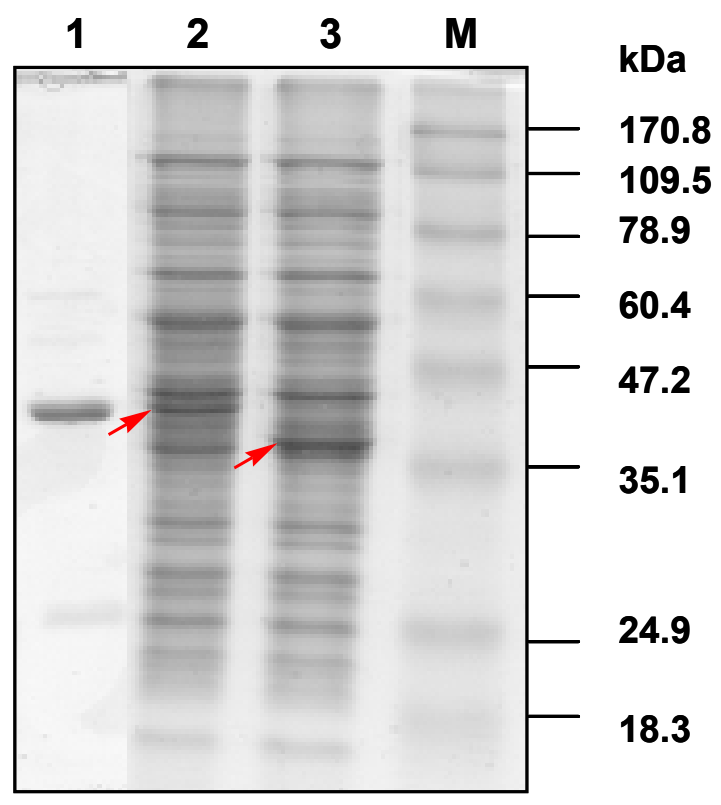

Figure S1. SDS-PAGE analysis of the overproduction and purification of AveBI from Streptomyces lividans

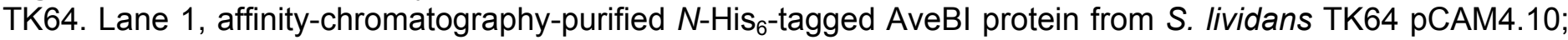
lane 2, soluble fractions from crude extracts of S. lividans TK64 pCAM4.10 expressing N-His 6 -tagged AveBI; lane 3, soluble fractions from crude extracts of S. lividans TK64 pCAM4.11 expressing native AveBI; lane M, protein molecular weight standard markers from Invitrogen (Carlsbad, CA). The expressed proteins are marked with arrows and molecular weights are indicated in the right column. 

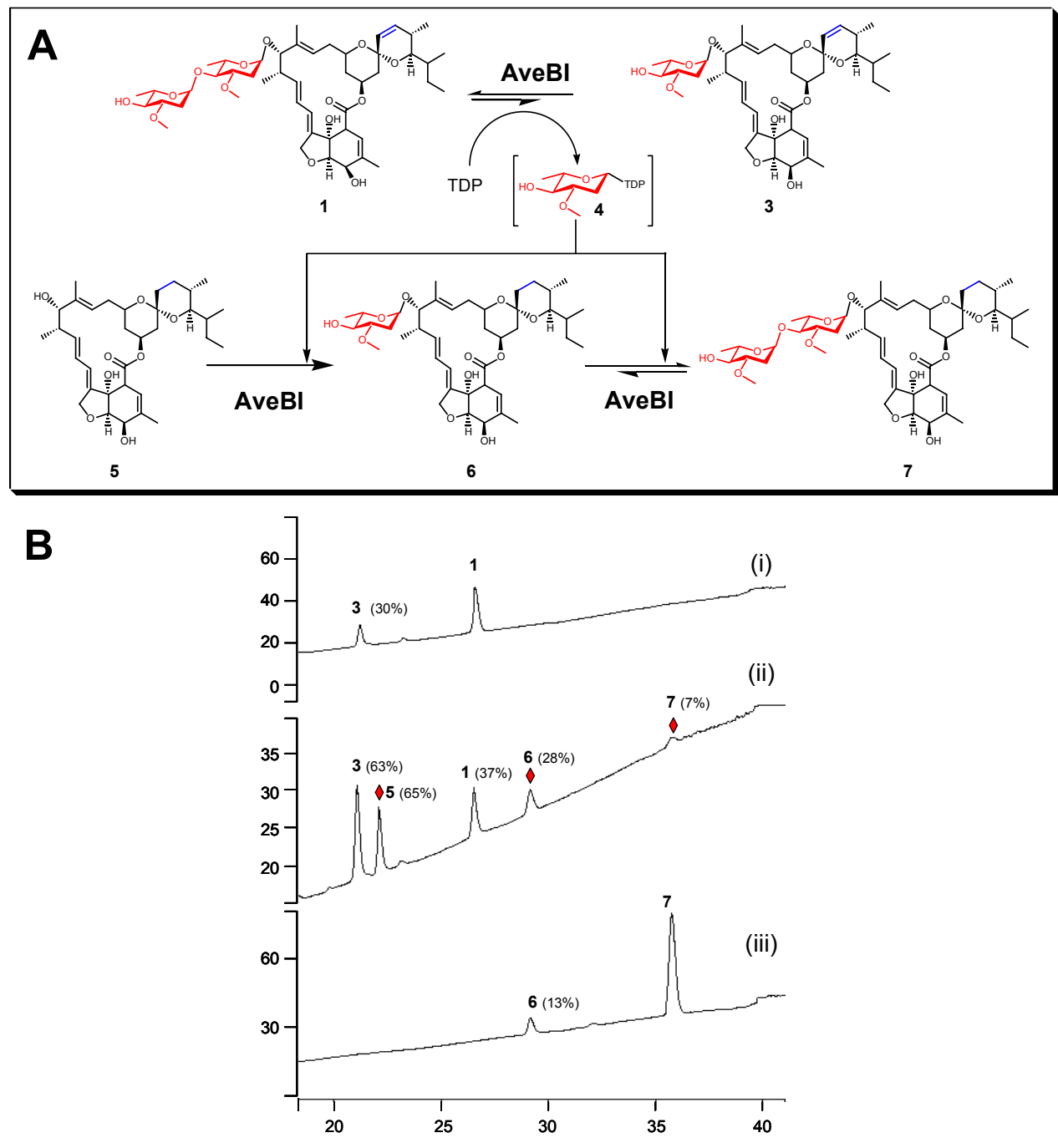

Figure S2. Representative AveBI-catalyzed aglycon exchange reactions. (A) Scheme for a AveBI-catalyzed aglycon exchange reaction. In this reaction, TDP-oleandrose (4) was excised from 1 by AveBI and subsequently transferred to $\mathbf{5}$, to produce $\mathbf{6}$ and $\mathbf{7}$ in a stepwise, tandem manner. (B) RP-HPLC analysis of AveBl-catalyzed reverse and aglycon exchange reactions. (i) Co-incubation of $100 \mu \mathrm{M} A \mathrm{AM} B 1 \mathrm{a}(1, \mathrm{M}+\mathrm{Na}$ 895.2) and $2 \mathrm{mM}$ TDP in the presence of $12 \mu \mathrm{M}$ of AveBI yielded 3 (30\%, M+Na 751.0). (ii) Co-incubation of $100 \mu \mathrm{M}$ AVM B1a (1), $100 \mu \mathrm{M} 5$ and $2 \mathrm{mM}$ TDP in the presence of $12 \mu \mathrm{M}$ of AveBI yielded $3(63 \%)$ from 1, and subsequently, TDP-oleandrose produced in situ was transferred consecutively to 5 to yield 6 (28\%) and 7 (7\%). (iii) Co-incubation of $100 \mu \mathrm{M}$ IVM (7) and $2 \mathrm{mM}$ TDP in the presence of $12 \mu \mathrm{M}$ AveBI yielded 6 (13\%). 

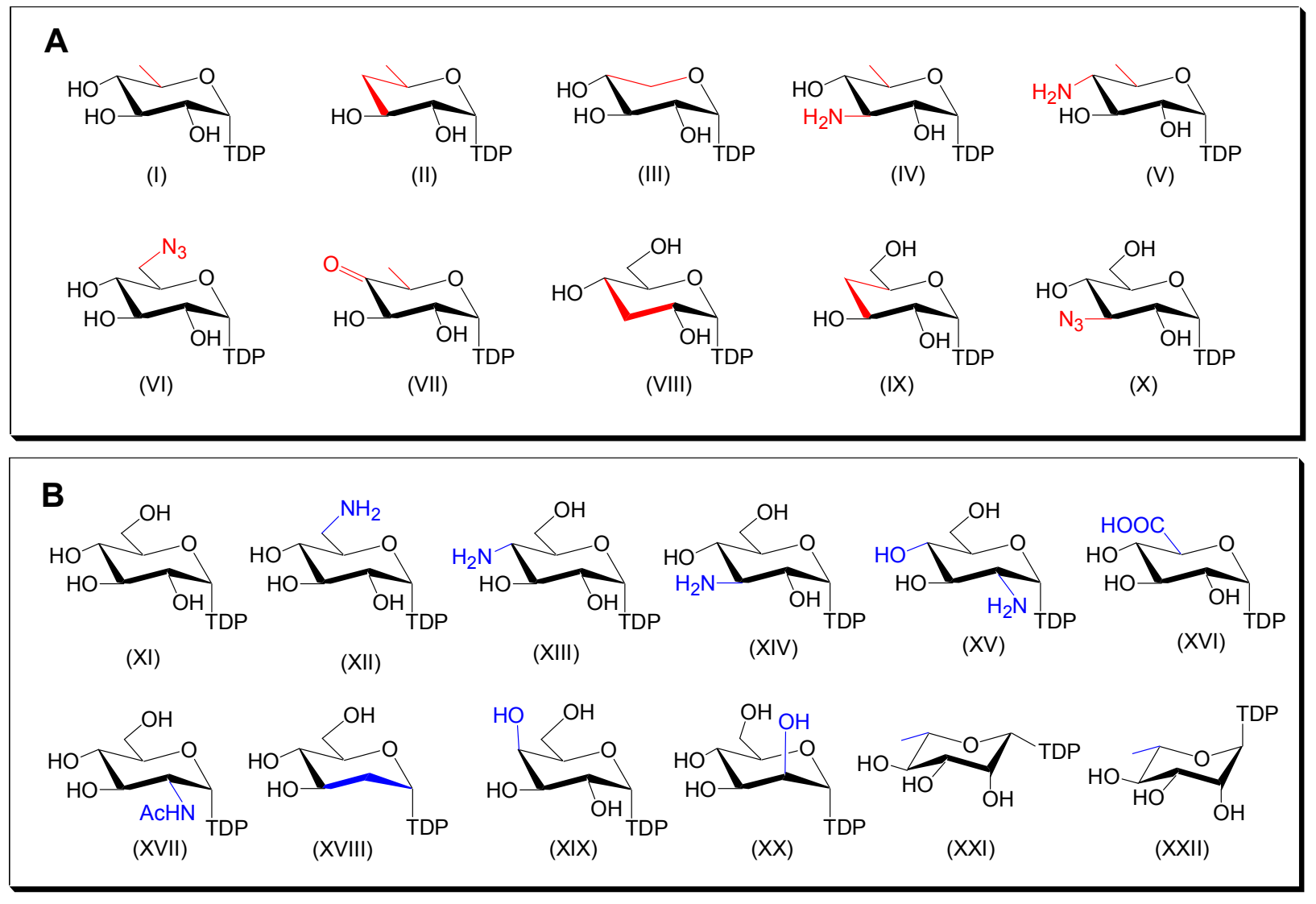

Figure S3. Structures of TDP-sugars tested in this work. The sugar donors in (A) were AveBI substrates while those in (B) were not. Highlighted parts (red or blue) indicate the structural differences from TDP- $\alpha-D-g l u c o s e$ (XI). 
Table S1. LC-MS characterization of AVM analogs.

\begin{tabular}{|c|c|c|c|c|c|c|}
\hline \multirow{2}{*}{$\begin{array}{c}\text { Compound } \\
\text { No. }^{a}\end{array}$} & \multirow{2}{*}{$\begin{array}{c}\text { Conversion } \\
\text { rate }(\%)^{b}\end{array}$} & \multirow{2}{*}{$\begin{array}{l}\text { Retention } \\
\text { time }(\min )^{c}\end{array}$} & \multicolumn{4}{|c|}{ MS $(m / z)$} \\
\hline & & & calcd & {$[\mathrm{M}+\mathrm{H}]^{+}$} & {$[\mathrm{M}+\mathrm{Na}]^{+}$} & {$[\mathrm{M}-\mathrm{H}]$} \\
\hline 2 & I & 16.6 & 584.3 & 585.2 & 607.0 & 584.3 \\
\hline $2 a$ & $94.4 \%$ & 12.3 & 730.4 & 731.2 & $\mathrm{nd}^{d}$ & 729.4 \\
\hline $2 b$ & $52.9 \%$ & 17.1 & 714.4 & $\mathrm{nd}^{d}$ & 737.2 & 713.4 \\
\hline $2 c$ & $28.9 \%$ & 10.5 & 716.4 & $\mathrm{nd}^{d}$ & 739.2 & 715.4 \\
\hline $2 d$ & $13.6 \%$ & 7.7 & 729.4 & 730.4 & 768.2 & 728.2 \\
\hline $2 e$ & $22.0 \%$ & 7.4 & 729.4 & 730.2 & 768.2 & 729.0 \\
\hline $2 f$ & $2.5 \%$ & 13.4 & 771.4 & 772.2 & 794.2 & 770.4 \\
\hline $2 \mathrm{~g}$ & $7.2 \%$ & 14.2 & 728.4 & 729.2 & 751.2 & 727.4 \\
\hline $2 \mathrm{~h}$ & $6.1 \%$ & 10.2 & 730.4 & $\mathrm{nd}^{d}$ & 753.4 & 729.4 \\
\hline $2 \mathbf{i}$ & $1.0 \%$ & 10.2 & 730.4 & $\mathrm{nd}^{d}$ & 753.4 & 729.4 \\
\hline $2 j$ & $1.0 \%$ & 13.2 & 771.4 & 772.2 & 794.2 & 770.4 \\
\hline 3 & I & 21.2 & 728.4 & 729.0 & 751.0 & 727.4 \\
\hline $3 a$ & $93.1 \%$ & 18.5 & 874.5 & 875.3 & 897.2 & 873.4 \\
\hline $3 b$ & $84.7 \%$ & 25.1 & 858.5 & 859.2 & 881.2 & 857.5 \\
\hline $3 c$ & $86.5 \%$ & 15.9 & 860.5 & 861.2 & 883.2 & 859.5 \\
\hline $3 d$ & $18.8 \%$ & 8.7 & 873.5 & 874.2 & $\mathrm{nd}^{d}$ & 872.4 \\
\hline $3 e$ & $19.9 \%$ & 8.2 & 873.5 & 874.0 & 896.2 & 872.5 \\
\hline $3 f$ & $2.4 \%$ & 20.3 & 915.5 & 916.0 & 938.0 & 914.4 \\
\hline $3 g$ & $6.7 \%$ & 23.7 & 872.5 & 873.2 & 895.0 & 871.5 \\
\hline $3 \mathrm{~h}$ & $8.0 \%$ & 14.6 & 874.5 & $\mathrm{nd}^{d}$ & 897.2 & 874.4 \\
\hline $3 \mathbf{i}$ & $5.7 \%$ & 14.6 & 874.5 & $\mathrm{nd}^{d}$ & 897.0 & 873.2 \\
\hline $3 \mathbf{j}$ & $2.5 \%$ & 18.3 & 915.5 & $\mathrm{nd}^{d}$ & 938.0 & 914.4 \\
\hline 5 & I & 22.1 & 586.4 & 587.2 & $\mathrm{nd}^{d}$ & 585.4 \\
\hline $5 a$ & $98.5 \%$ & 15.3 & 732.4 & 733.2 & 755.0 & 731.4 \\
\hline $5 b$ & $48.7 \%$ & 23.8 & 716.4 & 716.8 & 739.0 & 715.4 \\
\hline $5 c$ & $20.3 \%$ & 13.2 & 718.4 & 719.2 & $\mathrm{nd}^{d}$ & 717.4 \\
\hline $5 d$ & $20.8 \%$ & 8.7 & 731.4 & 732.0 & 754.0 & 730.4 \\
\hline $5 e$ & $24.4 \%$ & 8.1 & 731.4 & 732.0 & 754.0 & 730.4 \\
\hline $5 f$ & $9.6 \%$ & 21.4 & 773.4 & 774.2 & 796.0 & 772.4 \\
\hline $5 g$ & $22.4 \%$ & 18.8 & 730.4 & 731.2 & $\mathrm{nd}^{d}$ & 729.4 \\
\hline $5 \mathrm{~h}$ & $5.1 \%$ & 12.9 & 732.4 & $\mathrm{nd}^{d}$ & 755.0 & 731.4 \\
\hline $5 i$ & $1.6 \%$ & 14.2 & 732.4 & $\mathrm{nd}^{d}$ & 755.0 & 731.4 \\
\hline $5 j$ & $1.2 \%$ & 15.4 & 773.4 & $\mathrm{nd}^{d}$ & 796.6 & 772.4 \\
\hline
\end{tabular}


Table S1 (Continued)

\begin{tabular}{|c|c|c|c|c|c|c|}
\hline \multirow{2}{*}{$\begin{array}{c}\text { Compound } \\
\text { No. }^{a} \\
\end{array}$} & \multirow{2}{*}{$\begin{array}{c}\text { Conversion } \\
\text { rate (\%) }\end{array}$} & \multirow{2}{*}{$\begin{array}{c}\text { Retention } \\
\text { time }(\min )^{c}\end{array}$} & \multicolumn{4}{|c|}{ MS $(m / z)$} \\
\hline & & & calcd & ${ }_{[\mathrm{M}+\mathrm{H}]^{+}}$ & {$[\mathrm{M}+\mathrm{Na}]^{+}$} & {$[\mathrm{M}-\mathrm{H}]^{-}$} \\
\hline 6 & I & 29.5 & 730.4 & $\mathrm{nd}^{d}$ & 753.0 & 729.4 \\
\hline $6 a$ & $98.5 \%$ & 26.9 & 876.5 & $\mathrm{nd}^{d}$ & 899.0 & 875.4 \\
\hline $6 b$ & $85.2 \%$ & 34.4 & 860.5 & $\mathrm{nd}^{d}$ & 883.2 & 859.5 \\
\hline $6 c$ & $73.6 \%$ & 23.1 & 862.5 & $\mathrm{nd}^{d}$ & 885.0 & 861.4 \\
\hline $6 d$ & $18.2 \%$ & 8.1 & 875.5 & 876.2 & $\mathrm{nd}^{d}$ & 874.2 \\
\hline $6 e$ & $30.3 \%$ & 8.0 & 875.5 & 876.2 & 898.2 & 874.5 \\
\hline $6 f$ & $5.1 \%$ & 28.8 & 917.5 & $\mathrm{nd}^{d}$ & 940.2 & 916.4 \\
\hline $6 \mathrm{~g}$ & $18.7 \%$ & 33.1 & 874.5 & $\mathrm{nd}^{d}$ & 897.2 & 873.5 \\
\hline $6 h$ & $24.9 \%$ & 20.6 & 876.5 & $\mathrm{nd}^{d}$ & 899.2 & 875.4 \\
\hline $6 i$ & $9.2 \%$ & 20.7 & 876.5 & $\mathrm{nd}^{d}$ & 899.0 & 875.2 \\
\hline $6 j$ & $5.4 \%$ & 25 & 917.5 & $\mathrm{nd}^{d}$ & 940.0 & 916.4 \\
\hline 8 & I & 26.1 & 716.4 & $\mathrm{nd}^{d}$ & 739.2 & 715.4 \\
\hline $8 a$ & $95.3 \%$ & 23.5 & 862.5 & $\mathrm{nd}^{d}$ & 885.2 & 861.4 \\
\hline $8 b$ & $90.5 \%$ & 31.6 & 846.5 & $\mathrm{nd}^{d}$ & 869.2 & 845.2 \\
\hline $8 c$ & $80.8 \%$ & 20.2 & 848.5 & $\mathrm{nd}^{d}$ & 871.2 & 847.4 \\
\hline $8 d$ & $27.4 \%$ & 9.5 & 861.5 & 862.2 & 884.2 & 860.4 \\
\hline $8 e$ & $17.8 \%$ & 8.8 & 861.5 & 862.2 & 884.2 & 860.4 \\
\hline $8 f$ & $5.1 \%$ & 25.6 & 903.5 & $\mathrm{nd}^{d}$ & 926.2 & 902.4 \\
\hline $8 g$ & $7.0 \%$ & 28.4 & 860.5 & 861.2 & 883.2 & 859.5 \\
\hline $8 \mathrm{~h}$ & $4.4 \%$ & 18.0 & 862.5 & $\mathrm{nd}^{d}$ & 885.2 & 861.5 \\
\hline $8 i$ & $11.6 \%$ & 18.1 & 862.5 & $\mathrm{nd}^{d}$ & 885.4 & 861.4 \\
\hline $8 \mathbf{j}$ & $6.2 \%$ & 22.2 & 901.5 & 902.2 & 924.4 & 900.4 \\
\hline
\end{tabular}

${ }^{a}$ Compound numbering corresponds to Scheme $1 .{ }^{b}$ Percent conversions were determined by HPLC and calculated by dividing the integrated area of glycosylated product by the sum of the integrated area of the product plus the integrated area of the remaining substrate. 'HPLC parameters are described in 'Materials and Methods'. 'Not detected. 
Table S2. HRMS data for compounds in Table S1.

\begin{tabular}{|c|c|c|}
\hline Compound No. ${ }^{a}$ & $\begin{array}{c}\text { Calculated } \\
\text { Formula }[\mathrm{M}+\mathrm{Na}]^{+}\end{array}$ & $\begin{array}{c}\text { Observed } \\
{[\mathrm{M}+\mathrm{Na}]^{+}}\end{array}$ \\
\hline $2 a$ & 753.3821 & 753.3844 \\
\hline $2 b$ & 737.3871 & 737.3920 \\
\hline $3 b$ & 881.4658 & 881.4706 \\
\hline $3 c$ & 883.4450 & 883.4405 \\
\hline 5 & 609.3398 & 609.3450 \\
\hline $5 a$ & 755.3977 & 755.3987 \\
\hline $5 b$ & 739.4028 & 739.3994 \\
\hline 6 & 753.4184 & 753.4162 \\
\hline $6 a$ & 899.4763 & 899.4767 \\
\hline $6 b$ & 883.4814 & 883.4872 \\
\hline $6 c$ & 885.4607 & 885.4554 \\
\hline $8 a$ & 885.4607 & 885.4613 \\
\hline $8 b$ & 869.4658 & 869.4665 \\
\hline $8 c$ & 871.4450 & 871.4482 \\
\hline
\end{tabular}

${ }^{a}$ Compound numbering corresponds to Scheme 1. 